\title{
PRIMEROS DATOS SOBRE EL USO DE RECURSOS LEÑOSOS DURANTE EL HOLOCENO TEMPRANO EN EL CANAL BEAGLE: ESTUDIO ARQUEOBOTÁNICO DEL SITIO IMIWAIA I (TIERRA DEL FUEGO, ARGENTINA) \\ LAURA CARUSO FERMÉa ${ }^{\text {a }}$ A. FRANCISCO ZANGRANDO ${ }^{b}$ \& ERNESTO L. PIANA
}

\begin{abstract}
RESUMEN
Este trabajo tiene por objetivo estudiar el uso de los recursos vegetales leñosos llevado a cabo por sociedades cazadoras-recolectoras que habitaron el sitio Imiwaia I (costa norte del canal Beagle) durante el Holoceno temprano. Los resultados alcanzados indican que las sociedades cazadoras-recolectoras del sur de Tierra del Fuego hicieron un uso heterogéneo y local del material leñoso utilizado como combustible. Se utilizaron especies arbóreas y arbustivas, siendo Nothofagus pumilio y Berberis sp. los taxones con mayor representación.
\end{abstract}

PALABRAS CLAVE: arqueobotánica, Holoceno temprano, cazadores-recolectores, canal Beagle

\section{FIRST DATA ABOUT THE USE OF WOODEN RESOURCES DURING THE EARLY HOLOCENE IN THE BEAGLE CHANNEL ESTUDIO ARQUEOBOTÁNICO DEL SITIO IMIWAIA I (TIERRA DEL FUEGO, ARGENTINA)}

\begin{abstract}
This paper aims to study the use of wooden plant resources by hunter-gatherer societies that inhabited the northern coast of the Beagle channel site during the early Holocene. The results show the use of local and diverse wood as fuel in a southern site of Tierra del Fuego (Imiwaia I archaeological site). Both tree and shrub resources were used, being Nothofagus pumilio and Berberis sp. the most represented taxa.

KEY WORDS: archaeobotany, early Holocene, hunter-gatherers, Beagle channel

a Instituto Patagónico de Ciencias Sociales y Humanas (IPCSH-CONICET). Bv. Almirante Brown s/n (9120) Puerto Madryn, Chubut (Argentina). lcarusoferme@gmail.com

b Centro Austral de Investigaciones Científicas (CADIC - CONICET). Bernardo Houssay 200 (V9410CAB) Ushuaia, Tierra del Fuego (Argentina). panchozan@yahoo.com.ar

c Universidad Nacional de Tierra del Fuego (UNTDF). Onas 450 (CP9410) Ushuaia, Tierra del Fuego (Argentina). arqueologiatierradelfuego@gmail.com
\end{abstract}




\section{INTRODUCCIÓN}

En Tierra del Fuego las estrategias de gestión y uso de los recursos vegetales leñosos como combustible son hasta el momento conocidas para los grupos de cazadores-recolectores maritimos que ocuparon la costa sur de Tierra del Fuego durante el Holoceno tardío (Piqué i Huerta, 1999) y para cazadores recolectores el centro de la isla a principios del siglo XX, sitios Ewan I y Ewan II (Caruso Fermé et al. 2008; Caruso Fermé, 2010).

Los análisis arqueobotánicos llevados a cabo en diferentes sitios del canal Beagle (Túnel VII, Shamakush I y X, Alashauaia y Lanashuaia) correspondientes al Holoceno tardío mostraron una selección significativamente mayor de Nothofagus pumilio y Nothofagus betuloides sobre otras especies vegetales recolectadas de manera variable: Maytenus magellanica, Drimys winteri y Berberis sp. (Piqué 1999, pp. 286-287). Estos estudios condicen bien con las reconstrucciones paleoambientales efectuadas para la región principalmente a partir de registros polínicos, los cuales indican un paisaje de bosque cerrado de Nothofagus para el Holoceno tardío (Heusser, 1989, 1998, 2003). Para momentos más tempranos de la secuencia arqueológica del canal Beagle aún no se disponen de estudios arqueobotánicos.

Los estudios efectuados por Heusser (1989, 1993a, 1994) indican que las condiciones paleoambientales y la vegetación de la costa sur de Tierra del Fuego durante el Holoceno temprano y medio fueron distintas a las indicadas para momentos tardíos. Esto plantea que la disponibilidad de materiales leñosos habría sido diferente para el Holoceno temprano y con ello las decisiones humanas para la búsqueda y adquisición de estos recursos. Las investigaciones arqueológicas desarrolladas en la región del canal Beagle permitieron identificar ocupaciones de cazadores-recolectores con cronologías anteriores a los 6.400 años $\mathrm{AP}^{1}$ (Piana et al. 2012). El estudio de las mismas posibilitaría ampliar los trabajos arqueobotánicos desarrollados en el área para las ocupaciones correspondientes al Holoceno tardío (Piqué, 1999).

El objetivo de este trabajo es presentar los primeros datos sobre el uso de los recursos leñosos por sociedades cazadoras-recolectoras que habitaron la costa norte del canal Beagle durante el Holoceno temprano. Con este propósito fueron estudiados todos los restos de madera carbonizada recuperados en la capa $S$ del sitio arqueológico Imiwaia I, ubicado en la bahía Cambareces Interior -Tierra del Fuego, Argentina- (Piana et al. 2012). A partir de esta información se dan a conocer las especies vegetales utilizadas y se discute el papel que jugaron estos recursos para las sociedades tempranas de Tierra del Fuego.

Vegetación actual y su

evolución durante el Holoceno

La flora actual de la costa sur de la Isla Grande de Tierra del Fuego -hasta los 500-600 msnm- está dominada por el bosque Magallánico, interrumpido únicamente por turbales en los sectores más deprimidos de los valles (Moore, 1983). En esta asociación florística la diversidad de especies arbóreas es baja: entre ellas dominan dos especies de Nothofagus. En la porción occidental de la isla son más abundantes los Nothofagus betuloides -coihues o guindos- : árboles de hojas perennes, que alcanzan los 30 metros de altura y forman bosques cerrados. Este bosque es conocido como bosque mixto deciduosiempreverde. En el sector central de la margen sur de la isla, Nothofagus betuloides se mezcla con Nothofagus pumilio (lenga), de dimensiones similares pero de hoja caduca. Este sector se define como bosque deciduo o caducifolio con $N$. pumilio como taxón dominante y un sotobosque escaso en especies. Finalmente, en el extremo oriental fueguino (Península Mitre) vuelve a estar presente el bosque mixto deciduo-siempreverde. Esta variación en la composición del bosque obedece mayormente a la intensidad de las precipitaciones y a las condiciones de humedad de los suelos, en ambos casos mayores en la franja occidental del archipiélago fueguino (Tuhkanen 
et al. 1989-1990; Tuhkanen, 1992). También están presentes árboles de menor tamaño, como Drimys winteri (canelo) y Maytenus magellanica (maitén), especialmente en los sectores más húmedos. Bordeando los bosques prosperan Embothrium coccineum (notro) y arbustos como Berberis buxifolia (calafate) y Junellia tridens (mata negra). También existe una interesante diversidad de plantas rastreras, hongos, musgos y hepáticas (Moore, 1983).

Para los primeros cuatro milenios del Holoceno los espectros polínicos señalan la presencia de comunidades vegetaciones del ecotono bosque/ estepa, vinculada a un bosque abierto de Nothofagus en las costas del canal Beagle (Heusser, 1989; Ponce et al. 2011). Durante el Holoceno medio, a partir de los 6.000 años AP, con el incremento de la humedad y descenso de la temperatura se registra el desarrollo de un bosque denso y cerrado de Nothofagus (Heusser, 1998, p. 298), extendiéndose no sólo a lo largo de todo el canal Beagle sino también en los valles tributarios de los andes fueguinos (Heusser \& Coronato, 1989; Heusser \& Rabassa, 1987; Heusser, 2003; Borromei et al. 2007; Ponce et al. 2011). La condiciones de bosque cerrado se habrían mantenido hasta la actualidad, exceptuando un corto período entre 1.000 y 5.00 años AP en coincidencia con el Óptimo Climático Medieval, durante el cual se observa una reducción del bosque (Ponce et al. 2011).

\section{EL SITIO IMIWAIA I}

El sitio Imiwaia I se ubica dentro de un campo de drumlins asignado al Último Máximo Glacial (Rabassa et al. 1990), en un sector del canal Beagle que actualmente presenta costas recortadas y bahías poco profundas y angostas (Fig. 1). Este sitio arqueológico situado a $5 \mathrm{msnm}$ presenta evidencias de ocupaciones de cazadoresrecolectores adaptados al ambiente marino durante el Holoceno medio y tardío (Orquera \& Piana, 1999, 2009; Zangrando, 2009).

Investigaciones más recientes en Imiwaia I han proporcionado evidencia de ocupaciones cazadorasrecolectoras durante el Holoceno temprano (Piana et al. 2012). En la capa S -estrato basal del sitiose registró una ocupación con una cronología de $7.842 \pm 53$ años AP (8.420-8.662 cal. AP; Calib 6.0, $2 \partial$ curva SHCal04, McCormac et al. 2004). Dicho estrato tiene una potencia que varía entre los 6 y $12 \mathrm{~cm}$ en toda la extensión excavada $\left(80 \mathrm{~m}^{2}\right)$. En

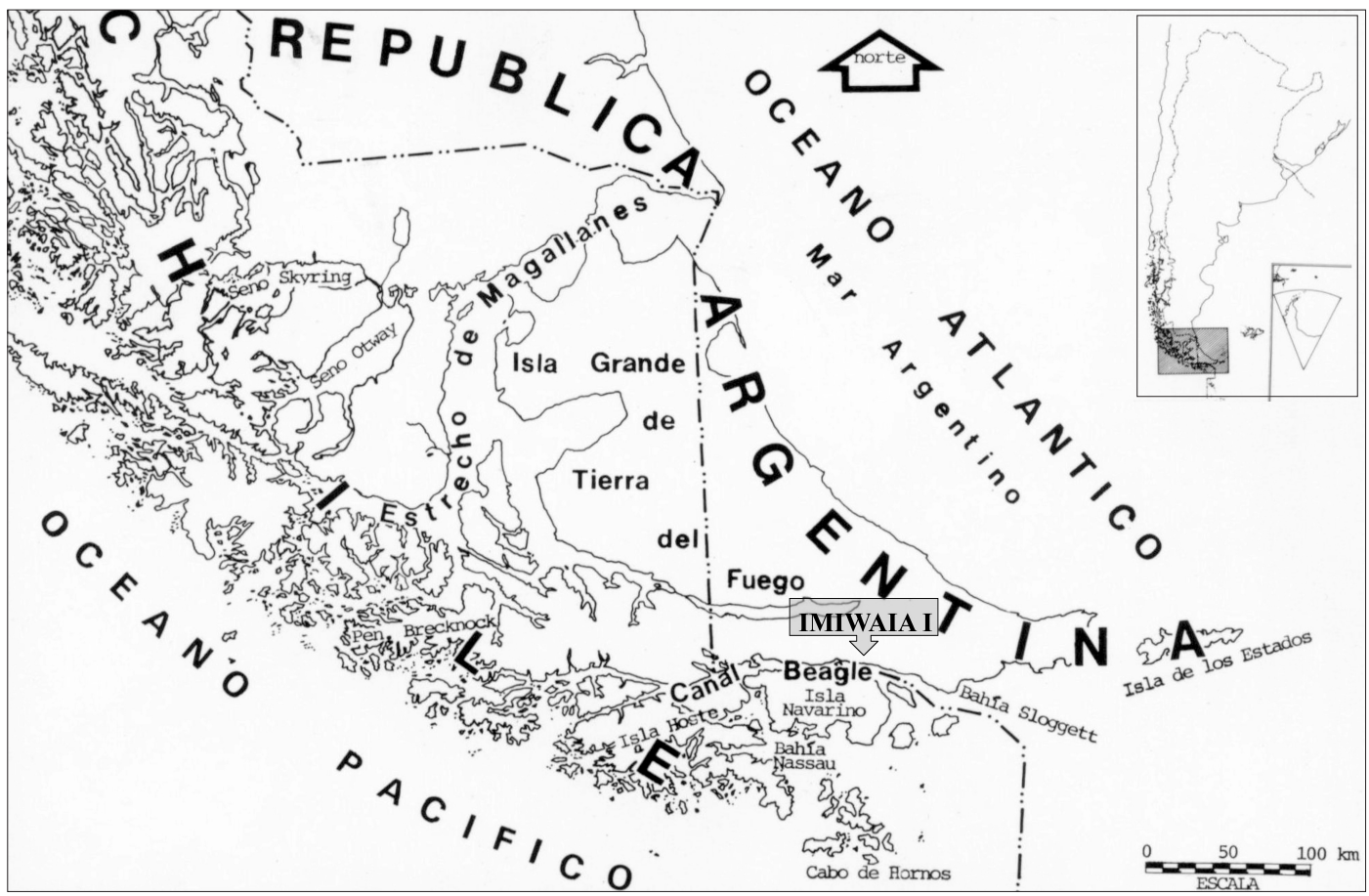

Fig. 1. Localización del sitio Imiwaia I. 
la cuadrícula $\mathrm{X}$ se registró un rasgo de combustión de aproximadamente $60 \mathrm{~cm}$ de diámetro y $3 \mathrm{~cm}$ de potencia, con huesos calcinados y una estructura de combustión de $35 \mathrm{~cm}$ de diámetro y $4 \mathrm{~cm}$ de profundidad, que atravesaba el cuerpo y la base de la capa, con guijarros termoalterados (Fig. 2). Cabe mencionar que la totalidad del material leñoso de la capa $\mathrm{S}$ procede de los rasgos de combustión antes descriptos. A pesar que en las cuadrículas VII y X se recuperaron una importante cantidad de lascas, microlascas y artefactos formatizados (Piana et al. 2011) no se registraron carbones dispersos u otros restos orgánicos.

\section{MATERIALES Y MÉTODOS}

La recuperación del material vegetal se realizó mediante el tamizado en seco del sedimento de la excavación. El tamaño de malla utilizada en los tamices fue de 1,5 mm. Este tipo de muestreo fue aplicado en forma rigurosa y sistemática en toda la excavación del depósito. La totalidad del material arqueobotánico existente en la capa S fue recuperado y estudiado.

El estudio arqueobotánico consistió en la determinación taxonómica de los carbones recuperados y en la identificación y caracterización

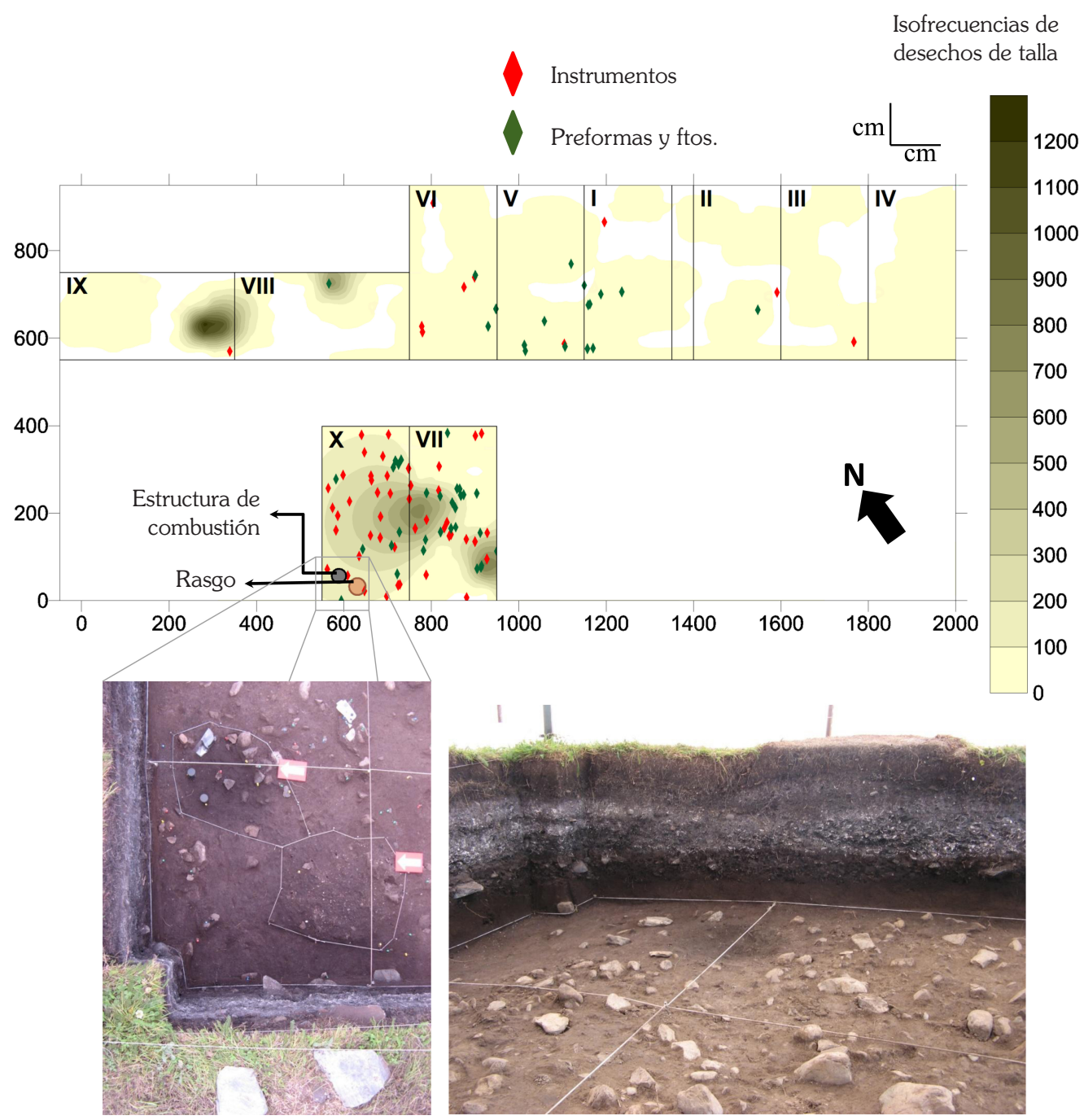

Fig. 2. Distribución y frecuencia de artefactos en la capa S de Imiwaia I con localización del rasgo y estructura de combustión: la imagen inferior izquierda muestra un detalle con la delimitación de estos sectores con restos de carbones; en la imagen inferior a la derecha se observa la profundización del rasgo en el till. 
de las alteraciones presentes en los mismos. El análisis de las alteraciones anatómicas de la madera permite, por un lado, estudiar las distintas modalidades de adquisición del material leñoso y, por otro, comprender la transformación de este recurso (Caruso Fermé, 2008, 2012; Euba et al. 2010; Schweingruber, 1996; Schweingruber et al. 2008; Théry-Parisot, 1998, etc.).

La identificación de la madera se llevó a cabo mediante la realización de cortes limpios de cada carbón, con el objetivo de obtener los tres planos anatómicos: transversal, longitudinal radial y longitudinal tangencial. Las muestras fueron visualizadas a través de un microscopio óptico de luz a reflexión de campo claro-oscuro Leica (MZ16A) y comparadas con muestras de referencia de madera actual y bibliografía especializada (Caruso Fermé, 2012, 2013, 2015; Solari, 1992).

\section{RESULTADOS}

El estudio arqueobotánico se basó en el análisis de la totalidad del material recuperado en la capa S, 146 fragmentos de madera carbonizada. El nivel de determinación ha sido mayoritariamente a nivel de especie; no obstante en algunos casos la poca variabilidad entre las especies de algunos géneros no permitió avanzar en su determinación.

El análisis taxonómico posibilitó la identificación de cinco taxones. Tres de los cuales corresponden a especies arbóreas: Nothofagus pumilio, Embothrium coccineum y Nothofagus sp. y dos arbustivas: Berberis sp. y Empetrum rubrum (Alberdi \& Donoso, 2004; Donoso, 1974; Donoso \& Donoso, 2006; Esconbar et al. 2006; Guerrido \& Fernández, 2007).

La similitud de las características anatómicas microscópicas de la madera de las especies pertenecientes al género Berberis hace difícil su identificación a nivel microscópico, por esta razón la identificación se realizó en el nivel de género (Caruso Fermé, 2012, 2015; Caruso Fermé et al. 2011).

De los cinco taxones identificados, Berberis sp. evidencia el porcentaje más alto $(54,93 \%)$, seguido por Nothofagus pumilio $(35,21 \%)$ y Embothrium coccineum $(8,45 \%)$ respectivamente. En el caso de Empetrum rubrum y Nothofagus sp., ambas se encuentran representadas por un solo fragmento de carbón (Tabla 1).
Tabla 1. Restos de carbón procedentes de la capa S, sitio Imiwaia I, por taxón.

\begin{tabular}{|c|c|c|}
\hline \multicolumn{3}{|l|}{ Sitio IMIAWAIA I (Capa S) } \\
\hline Taxón & $\mathrm{N}$ & $\%$ \\
\hline Berberis sp. & 78 & 54,93 \\
\hline Nothofagus pumilio & 50 & 35,21 \\
\hline Embothrium coccineum & 12 & 8,45 \\
\hline Nothofagus sp. & 1 & 0,70 \\
\hline Empetrum rubrum & 1 & 0,70 \\
\hline Total restos identificados & 142 & 100 \\
\hline Número de taxa & & 5 \\
\hline Indeterminables & & 4 \\
\hline
\end{tabular}

El estudio arqueobotánico de los carbones posibilitó el registro de dos tipos de alteraciones: por microorganismos (hifas/micelio) y vitrificación. No obstante, el número de fragmentos afectados es sumamente bajo. Dos carbones de Nothofagus pumilio presentan hifas y el carbón de Empetrum rubrum indicios de vitrificación. La presencia de nudos en tres carbones y un alto grado de vitrificación en uno de los fragmentos imposibilitó la determinación de estos cuatro fragmentos.

\section{DISCUSIÓN}

Los resultados alcanzados, a partir del estudio arqueobotánico de la madera carbonizada procedente del sitio Imawaia I (capa S), permitieron ampliar el conocimiento sobre las gestión de los recursos vegetales por parte de las sociedades cazadoras-recolectoras que habitaron la región del canal Beagle durante el Holoceno temprano

El estudio de restos vegetales es considerado una herramienta útil para comprender aspectos tales como la subsistencia, la movilidad y organización de un grupo (Pearsall, 1989; Popper \& Hastorf, 1988). El análisis taxonómico de la madera carbonizada procedente del rasgo $y$ de la estructura de combustión de la capa $\mathrm{S}$ de Imiwaia I permitió determinar cinco taxones: Nothofagus pumilio, Embothrium coccineum, Empetrum rubrum, Berberis sp. y Nothofagus sp. Los resultados del análisis demuestran un uso heterogéneo y local del material leñoso destinado a la combustión. Si bien no es posible asumir que todos los restos arqueobotánicos procedan de los 
alrededores del sitio, la recolección de combustible -dado su uso cotidiano- puede ser desarrollada en el ámbito local, en un radio no muy alejado de los lugares de habitación (Caruso Fermé, 2015). A partir de estudios etnográficos se puede constatar que prácticamente todos los grupos humanos seleccionan los combustibles en función de factores tales como las propiedades de las maderas, la disponibilidad o normas culturales (Smart \& Hoffman, 1988; Shackleton \& Prins, 1992; Zapata, 1999). La morfología de la madera también puede condicionar su selección, ya que por ejemplo las ramas pequeñas pueden servir como iniciadores de fuego mientras que las piezas más grandes como combustible propiamente dicho (Caruso Fermé, 2012). Los análisis arqueobotánicos llevados a cabo por Piqué (1999) en la costa norte del canal de Beagle (sitios Tunel VII, Lanashuaia, Alashawaia y Shamakush I y X) sugieren para la autora un uso preferencial de aquellos taxones más abundantes durante el Holoceno tardío, como es el caso de Nothofagus pumilio y Nothofagus betuloides, acompañados de otros menos abundantes tales como Berberis sp. y Maytenus magellanica. Según Piqué las propiedades físicoquímicas de estas especies harían que fueran consideradas buenos combustibles (Piqué, 1999, pp. 286-287).

El análisis de la madera carbonizada de Imiwaia I (capa S) permitió a su vez determinar la presencia de dos tipos de alteraciones entre los carbones estudiados: alteración por microorganismos (hifas/micelio) y vitrificación. La primera de ellas representada en dos carbones de Nothofagus pumilio y la segunda en un fragmentos de Empetrum rubrum. El análisis de las alteraciones permite, entre otras cosas, estudiar las distintas modalidades de adquisición del material leñoso, por ejemplo el estado de la madera utilizada como combustible. La diferenciación de la madera sana y la madera alterada permite distinguir una recolección de tipo aleatoria fundada en la accesibilidad de la madera, en la cual se privilegia una elección basada en el estado fenomenológico, fisiológico y morfológico de la madera; de una recolección de tipo selectiva basada en la gestión de la madera en pie (Thèry-Parisot, 1998). Por lo tanto, la baja presencia de carbones con hifas o micelios y la ausencia de carbones con marcas de insectos xilófagos podrían sugerir que el material leñoso utilizado como combustible durante la ocupación de la capa S de Imiwaia se encontraba en buen estado.

Estudios paleoambientales realizados en la costa norte del canal Beagle postulan que para el 8.000 años AP esta zona se caracterizaba por un tipo de vegetación de bosque abierto (Heusser, 2003; Markgraf, 1980, 1983; Rabassa et al. 1989). Las especies representadas en la capa S de Imiwaia I sugieren que la obtención del material leñoso podría haberse dado en formaciones boscosas de este tipo. Es importante destacar que tanto Empetrum rubrum como Berberis sp. habitan en los bordes y zonas abiertas del bosque así como también en zonas de estepa, donde Berberis sp. desarrolla mayor tamaño (Correa, 1998; Guerrido \& Fernández, 2007). Por lo tanto ambas especies podrían ser indicativas de la existencia de espacios abiertos del bosque y/o zonas esteparias. Los diagramas polínicos realizados por Heusser (1989, 1993b, 1994) no evidencian la presencia de Berberis sp. en la costa norte del canal Beagle para la época de ocupación documentada en Imiwaia. No obstante, cabe tener presente que las muestras palinológicas y las antracológicas reflejan escalas espaciales diferentes (Allué, 2002; Zapata, 2007). A diferencia de las muestras de carbón o madera arqueológica, producto de la actividad humana, el polen puede tener un componente regional y también evidenciar una vegetación de un ámbito espacial mayor (Zapata, 2007). Además el modo de dispersión y la diferente producción del polen de las distintas plantas pueden determinar la importancia relativa, la sobrerrepresentación, así como también la ausencia de algunos taxones (Moore et al. 1991). Por ello no siempre es posible realizar comparaciones directas entre los registros polínicos y antracológicos. En efecto, a pesar que los resultados obtenidos para la capa $S$ de Imiwaia I permiten señalar una representación similar de las comunidades vegetales del Holoceno temprano a la de los registros polínicos, esta interpretación debe ser tomada con cautela por varias razones. Si bien la procedencia de la muestra (carbones concentrados) y los estudios polínicos permiten 
conocer la disponibilidad de los recursos leñosos, presentan ciertas limitaciones a la hora de poder cuantificar la biomasa característica del ambiente en momentos de la ocupación de un sitio (Zapata, 1999).

\section{CONCLUSIONES}

El estudio de la madera carbonizada procedente de la capa $\mathrm{S}$ del sitio Imiwaia I permitió obtener información sobre el uso de los recursos vegetales leñosos por parte de los grupos cazadores-recolectores de la costa norte del canal de Beagle durante el Holoceno temprano. Los resultados alcanzados posibilitaron también ampliar los estudios arqueobotánicos en esta región, dado que hasta el momento los trabajos realizados corresponden únicamente a ocupaciones del Holoceno tardío.

Los resultados aquí presentados indican que los grupos cazadores recolectores que ocuparon el sitio Imiwaia I, durante el Holoceno temprano, hicieron un uso heterogéneo y local del material leñoso utilizado como combustible. Entre los restos estudiados se determinaron especies arbóreas (Nothofagus pumilio, Nothofagus sp. y Embothrium coccineum) y arbustivas (Empetrum rubrum y Berberis sp.), siendo Nothofagus pumilio y Berberis sp. los taxones con mayor representación. Ambas especies leñosas comprenden más del $90 \%$ de la muestra analizada.

Por lo que respecta al estado del material leñoso utilizado como combustible, la escasa evidencia de alteraciones en los carbones estudiados sugiere que la madera se encontraba en buen estado antes de su combustión.

La consonancia entre los resultados arqueobotánicos alcanzados y los registros polínicos demuestran que las especies leñosas utilizadas como combustible estaban disponibles en las inmediaciones del sitio Imiwaia en los momentos de ocupación. Si bien no es factible emplear esta información para inferir la oferta vegetal o biomasa de taxones, los resultados señalados condicen bien con las reconstrucciones paleoambientales para la región, las cuales indican condiciones de bosque abierto o ecotono de bosque/estepa durante el Holoceno temprano.

\section{AGRADECIMIENTOS}

Estas investigaciones están siendo financiadas por el proyecto PIP 0387 (CONICET). Agradecemos al Departament d'Arqueología i Antropología de la Institución Milà y Fontanals del Centro Superior de Investigaciones Científicas (CSIC-Barcelona) por proporcionar el instrumental necesario para el desarrollo del estudio arqueobotánico presentado en este trabajo. A Timothy Jull y Mitzi De Martino (Arizona AMS Laboratory) por su respaldo en los análisis radiocarbónicos.

Queremos agradecer especialmente a la familia Goodall y a los integrantes de la Ea. Harberton por ofrecernos ayuda constante durante las actividades en el campo. Hein Bjerck, Heidi Breivik, María Filippelli, María Paz Martinoli, Maria José Saletta y Angélica Tivoli colaboraron en las tareas de excavación de la cuadrícula X de Imiwaia I.

\section{BIBLIOGRAFÍA}

Alberdi, M., \& Donoso, C. (2004). Variación en Nothofagus siempreverdes sudamericanos. En: Variación Intraespecífica en las especies arbóreas de los bosques templados de Chile y Argentina. Editorial Universitaria.

Allué, E. (2002). Dinámica de la vegetación y explotación del combustible leñoso durante el Pleistoceno Superior y el Holoceno del Noreste de la Península Ibérica a partir del análisis antracológico. Tesis doctoral. Tarragona, Universitat Rovira i Virgili.

Borromei, A. M., Coronato, A., Quattrocchio, M., Rabassa, J., Grill, S., \& Roig, C. (2007). Late Pleistocene-Holocene environments in Valle Carabajal, Tierra del Fuego, Argentina. Journal of South America Earth Sciences, 23, 321-355.

Caruso Fermé, L. (2008). Los usos de la madera entre los cazadores-recolectores Selknam de Tierra del Fuego. Treball de recerca-Doctorat d'Arquelogía Prehistòrica. Universitat Autónoma de Barcelona.

Caruso Fermé, L. (2010). Ethnographie, archéobotanique et expérimentation sur le site d'Ewan I (Tierra del Fuego, Argentine). Anthropobotanica $\mathrm{N}^{\circ}$ 1.5: 3-17. Muséum national d'Histoire naturelle, Paris. France.

Caruso Fermé, L. (2012). Modalidades de adquisición y usos del material leñoso entre grupos cazadoresrecolectores patagónicos (Argentina). Métodos y 
técnicas de estudio del material leñoso arqueológico. Tesis doctoral. Universitat Autònoma de Barcelona (UAB) España.

Caruso Fermé, L. (2013.) Los recursos vegetales en arqueología: estrategias de muestreo y estudio del material leñoso. Dunken. Buenos Aires, Argentina. ISBN: 978-987-026738-6.

Caruso Fermé, L. (2015). Modalidades de adquisición y usos de la madera en sociedades cazadoras-recolectoras patagónicas: métodos y técnicas de estudio. Treballs d'Etnoarqueología 10. Consejo Superior de Investigaciones Científicas. Madrid, España.

Caruso fermé L., Álvarez M., \& Vázquez M. (2011). Análisis arqueobotánico de piezas de madera del extremo austral americano. Magallania, 39(1), 221-240.

Correa M. N. (1998.) Flora Patagónica, Parte I. Colección Científica del INTA. Buenos Aires

Donoso, C. (1974). Dendrología: Árboles y Arbustos Chilenos. Universidad de Chile, Facultad de Ciencias Forestales, Manual No 2 .

Donoso, C., \& Donoso, P. (2006). Nothofagus betuloides (Mirb) Oerst. Coigüe de Magallanes, Ouchpaya onas), Guindo (zona Austral), Coigüe Colorado (Tierra del Fuego). En: Las especies arbóreas de los bosques templados de chile y argentina. Autoecología. 411423. Donoso editor

Escobar, B., Donoso, C., Souto, C., Alberdi, M., \& Zúñiga, A. (2006). Embothrium coccineum J. R. et G. Forster En: Las especies arbóreas de los bosques templados de Chile y Argentina. Autoecología. 233-245. Donoso editor

Euba Rementeria, I., Allué Marti, E., \& Palet Martínez J. M. (2010). Alteraciones Biológicas de la Madera: El Estudio de los Materiales constructivos del Yacimiento de Pleta De L'estall Serrer (Valle Del Madriu, Andorra). En: VIII Congreso Ibérico de Arqueometría- Sesión Biomateriales, pp. 1-10.Fischesser, B. (2000) El libro de El árbol (trans.) T. Almaraz. Madrid: Ed. Drac, edicion española.

Guerrido, C., \& Fernández, D. (2007). Flora Patagonia. Punta Arenas: Fantástico Sur.

Heusser C. J. (1989). Late Quaternary vegetation and climate of southern Tierra del Fuego. Quaternary Research, 31, 396-406.

Heusser, C. J. (1993a). Late Quaternary forest-steppe contact zone, Isla Grande de Tierra del Fuego, subantarctic South America. Quaternary Science. Reviews, 12, 169-177.

Heusser, C. J. (1993b). Paleoindians and fire during the late
Quaternary in southern South America. Revista Chilena de Historia Natural, 67, 435-443.

Heusser, C. J. (1994). Quaternary paleoecology of FuegoPatagonia. Revista del Instituto Geológico, 15, 7-26.

Heusser, C. J. (1998). Deglacial paleoclimate of the American sector of the Southern Ocean: late GlacialHolocene records from the latitude of Canal Beagle

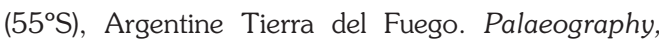
Palaeoclimatology, Palaeoecology, 141, 277-301.

Heusser, C. J. (2003). Ice Age Southern Andes. A Chronicle of Paleoecological Events. Developments in Quaternay Science 3. Elsevier, Amsterdam.

Heusser C. J., \& Coronato A. (1989). Peatbog accumulation rate in the Andes of Tierra del Fuego and Patagonia (Argentina and Chile) during the last 43,000 years. Pirineos, 133, 113-122.

Heusser, C. J., \& Rabassa, J. (1987). Cold climatic episode of Younger Dryas age in Tierra del Fuego. Nature, 328, 609-611.

Markgraf V. (1980). New data on the late and Postglacial vegetational history of La Misión, Tierra del Fuego, Argentina. Proc. IV Int. Palinology Conf., Lucknow, India, 3, 68-74.

Markgraf V. (1983). Late and Postglacial vegetational and paleoclimatic changes in subantarctic, temperate and arid environments in Argentina, Palynology, 7, 43-70.

McCormac F. G., Hogg, A. G., Blackwell, P. G., Buck, C. E., Higham, T. F. G., \& Reimer, P. J. (2004). SHCAL04 Southern Hemisphere Calibration, 0-11.0 CAL KYR BP. Radiocarbon, 46(3), 1087-1092.

Moore D. M. (1983). Flora of Tierra del Fuego. Anthony Nelson, Englan and Missouri Botanical Garden U.S.A

Moore, P. D., Webb, J. A., \& Collinson, M. E. (1991). Pollen analysis. Blackwell Scientific Publications. Oxford.

Orquera, L. A., \& Piana, E. L. (1999). Arqueología de la región del canal Beagle (Tierra del Fuego, República Argentina).Buenos Aires, Sociedad Argentina de Antropología.

Orquera, L. A., \& Piana, E. L. (2009). Sea nomads of the Beagle Channel in Southernmost South America: Over six thousand years of coastal adaptation and stability. Journal of Island \& Coastal Archaeology, 4, 61-81.

Pearsall, D. (1989). Paleoethnobotany. A Handbook of Procedures. San Diego: Academic Press.

Piana, E. L., Zangrando, A. F., \& Orquera, L. A. (2011). Cazadores-recolectores tempranos en la región del canal Beagle (Tierra del Fuego, Argentina): la evidencia de la capa S de Imiwaia I. Libro de resúmenes de las VIII Jornadas de Arqueología de la Patagonia: 65 65, 
Malargüe - Argentina.

Piana, E. L., Zangrando, A. F., \& Orquera, L. A. (2012). Early occupations in Tierra del Fuego and the evidences from $\mathrm{S}$ layer in Imiwaia I site (Beagle Channel, Argentina). Current Research in the Pleistocene, Center for the Study of First American. Southbound. Late Pleistocene Peopling of Latin America (Special edition), 171-175.

Piqué i Huerta, R. (1999). Producción y uso del combustible vegetal: una evaluación arqueológica. Traballs d'Etnoarqueología 3. Consejo Superior de Investigaciones Científicas, Madrid.

Ponce, J. F., Borromei, A. M., \& Rabassa J. O. (2011). Evolución del paisaje y de la vegetacion durante el cenozoico tardío en el extremo sureste del archipiélago fueguino y canal Beagle. En A. F. Zangrando, M. Vázquez y A. Tessone (Eds.), Los cazadores-recolectores del extremo oriental fueguino. Arqueología de Península Mitre e Isla de los Estados (pp. 31-64). Buenos Aires: Sociedad Argentina de Antropología.

Popper, V., \& Hastorf, C. (1988). Introduction. En Current Paleoethnobotany Analytical Methods and Cultural Interprtations of Archaeological Plant Remains. C. Hastorf y V. Popper (Eds.) (pp. 1-16). Chicago: The University of Chicago Press.

Rabassa, J., Heusser, C. J., \& Coronato, A. (1989). Peatbog accumulation rate in the Andes of Tierra del Fuego and Patagonia (Argentina y Chile) during the last 43.000 years. Pirineos, 133, 113-112.

Rabassa, J., Heusser, C., \& Rutter, N. (1990). Late Glacial and Holocene sea transgression in the Beagle Channel (Tierra del Fuego). Quaternary of South America and Antarctic Peninsula, 7, 335-360.

Schweingruber, F. H. (1996) Tree Ring and Environment Dendroecology. Berne, Stuttgart, Viena, Swiss Federal Institute for Forest, Snow and Landscape Research, WSL/FNP, Birnmensdorf.

Schweingruber, F. H., Börner, A., \& Schulze, E.-D. (2008). Atlas of Woody Plant Stems. Evolution, Structure, and Environmental Modifications. Springer-Verlag
Berlin Heidelberg

Shackleton, N. J., \& Prics, F. (1992) Charcoal analysis and the "Principle of Least Effort"- A Conceptual Model. Journal of Archaeological Science, 19, 631-637

Smart, T. L., \& Hoffman, E. S. (1988). Environmental Interpretation of Archaeologiacl Charcoal. En Hastorf \& Popper (Eds.), Currente Paleoethrobotany. Chicago y Londres. The University of Chicago Press 167-2015.

Solari, M. E. (1992) Anthracologie et ethnoarchéologie dans l'archipel du cap Horn (Chili). Bolletin de la Société Botanique de France, 139, 407-420.

Théry-Parisot, I. (1998). Économie du Combustible et Paléoécologie en contexte glaciaire et périglaciaire, Paléolithique moyen et supérieur du sud de la France. (Anthracologie, Expérimentation, Taphonomie). Thèse de doctorat. Université de Paris I PanthéonSorbonne.

Tuhkanen, S. (1992). The climate of Tierra del Fuego from a vegetation geographical point of view and its ecoclimatic counterparts elsewhere. Acta Botanica Fennica, 145, 1-64.

Tuhkanen, S., Koukka, I., Hyvönen, J., Stenroos, S., \& Niemelä, J. (1989-1990). Tierra del Fuego as a target for biogeographical research in the past and present. Anales Instituto de la Patagonia, Serie Ciencias Naturales, 19, 1-107.

Zangrando, A. F. (2009). Historia evolutiva y subsistencia de cazadores-recolectores marítimos de Tierra del Fuego. Buenos Aires: Sociedad Argentina de Antropología.

Zapata, L. (1999). El combustible y la agricultura prehistórica: Estudio arqueobotánico de los yacimientos de Arenaza, Kanpanoste Goikoa y Kobaederra. Cuadernos de prehistoria-arqueología, 10(S), 305-337.

Zapata, L (2007). Cazadores-recolectores y recursos vegetales. En C. Cacho, R. Maicas, J A. Martos, \& M. I. MartínezNavarrete (Coords.), Acercándonos al pasado. Museo Arqueológico Nacional. Madrid: CSIC. 
